\title{
Medication-related osteonecrosis of the jaw in patients with bone metastases from solid tumors: analysis of the Istituto Oncologico Veneto case series
}

Selma Ahcene-Djaballah ${ }^{1}$, stefania zovato, Christian Bacci ${ }^{2}$, Giordana Bettini ${ }^{3}$, MARCO MARUZZO, Francesca Di Sarra ${ }^{1}$, Alfonso Massimiliano Ferrara ${ }^{1}$, Antonella Brunello ${ }^{1}$, VALENTINA GUARNERI ${ }^{2}$, Evelina Lamberti ${ }^{1}$, CRISTINA Falci $^{1}$, Vittorina Zagonel ${ }^{1}$

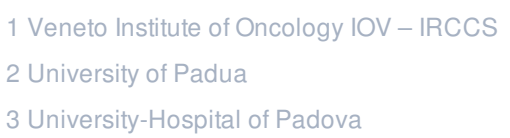

Funding: The author(s) received no specific funding for this work.

Potential competing interests: The author(s) declared that no potential competing interests exist.

\section{Abstract}

Treatment of BM bone metastases from solid tumors often includes the use of bone antiresorptive agents (BAA) beside tumor-directed specific treatment. Medication-related osteonecrosis of the jaw (MRONJ) is a severe side effect caused by BAA. This retrospective study evaluates the incidence of MRONJ in a real-world setting of patients with solid tumors treated with BAA for BM at Istituto Oncologico Veneto (IOV). Data for patients with solid tumors who received BAA for BM between January 2010 and December 2017 at Istituto Oncologico Veneto, Padua, Italy were retrieved from a prospectively maintained database, 1211 patients met the inclusion criteria and received BAA . In addition, we defines the time to onset (TTO) and assess the role of dental prevention.

Background: Bone metastases (BM) are responsible for high morbidity and limit the quality of life (QoL) of patients (1). Treatment of BM from solid tumors often includes the use of bone antiresorptive agents (BAA) beside tumor-directed specific treatment(2,3). Thanks to progress in available antitumor therapies, BAA are more and more used as long-term treatment. This may lead to an increase in the risk of medication related osteonecrosis of the jaw (MRONJ) which is a serious adverse event of BAA impacting QoL. In randomized clinical trials, MRONJ reported incidence ranged from $0.4 \%$ to $3.2 \%(4,5,6,7)$, yet estimated higher in observational studies $(\mathbf{5 , 8})$. The association between cumulative doses, duration of treatment and MRONJ incidence remains controversial, and there are open questions from clinical practice, such as the estimation of MRONJ risk for all the patients that switch BAA from bisphosphonates (BP) to denosumab (Dmb).

The aim of this study was to determine the incidence of MRONJ in a real-world setting of patients with solid tumors treated with BAA for BM at Istituto Oncologico Veneto (IOV). In addition, to define the time to onset (TTO) and assess the role of dental prevention.

Patients and methods Data for patients with solid tumors who received BAA for BM between January 2010 and December 2017 at Istituto Oncologico Veneto, Padua, Italy were retrieved from a prospectively maintained database. 
Patients who had received radiation therapy in head and neck region, and those with concomitant myeloma were excluded. Incidence of MRONJ and TTO were calculated from BAA start and the time of MRONJ diagnosis.

Results: Out of 1301 patients with BM from solid tumors, 1211 patients met the inclusion criteria and received either zoledronic acid (Zol) 961 (79.4\%) or Dmb 68 or both 182 (15\%). The malignancies most frequently associated with BM were breast cancer (487 patients, 40.2\%), lung cancer (224, 20\%), prostate cancer (172, 14\%), and gastrointestinal cancer (151, 13\%). Median age at BM diagnosis was 65 years (range 21-90), 59\% were female. Duration of BAA was less than 6 months in 481 patients (40\%), it was 6 to 24 months in 405 patients (33\%) and more than 24 months in 323 patients (23\%). Eighty-one patients developed MRONJ (6.7\%). The incidence of MRONJ in patients receiving Dmb or Zol was $4.4 \%$ and $6.4 \%$, respectively with incidence rising to 12.4 for patients who switched from Zol to Dmb, with a median TTO of 29.9 months (range 1.9-124.2). Proper measures regarding prevention of oral diseases dental issues before starting BAA were undertaken in $77.8 \%$ of patients, and dental screening procedures significantly reduced the risk of MRONJ ( $p=0.0093)$. After stopping BAA treatment due to MRONJ, 18 patients (22.2\%) experienced bone progressive disease, with 12 of them presenting a SRE.

Conclusion: Real-world data pointed to a risk of MRONJ higher than figures reported from clinical studies. Additional studies are warranted to clarify the pathological mechanisms of MRONJ, as well as to stratify risk factors and diverse patient populations and the strategies for MRONJ management along with "ideal timing" for oral health follow-up.

\section{References:}

1- Cecchini M, Wetterwald A, Pluijm G,et al. Molecular and biological mechanisms of bone metastasis. EAU Update Series 2005;3:214-26.

2-Van Poznak C, Somerfield MR, Barlow WE, et al. Role of bone-modifying agents in metastatic breast cancer: an American Society of Clinical Oncology-Cancer Care Ontario focused guideline update. J Clin Oncol. 2017;35(35):3978-3986.

3-Anderson K, Ismaila N, Flynn PJ, et al. Role of bone-modifying agents in multiple myeloma: American Society of Clinical Oncology clinical practice guideline update. J Clin Oncol. 2018;36(8):812-818.

4-Stopeck A.T. , Lipton A , Body J.J. et al.Denosumab compared with zoledronic acid for the treatment of bone metastases in patients with advanced breast cancer: a randomized, double-blind study J Clin Oncol, 2010, 28: 5132-5139;

5- Khan AA, Morrison A, Hanley DA, et al; International Task Force on Osteonecrosis of the Jaw. Diagnosis and management of osteonecrosis of the jaw: a systematic review and international consensus. J Bone Miner Res. 2015;30(1):3-23.

6-Henry, D..; Costa, L.; Goldwasser, F.; et al. Randomized, double-blind study of denosumab versus zoledronic acid in the treatment of bone metastases in patients with advanced cancer (excluding breast and prostate cancer) or multiple myeloma.J. Clin. Oncol.2011,29, 1125-1132.

7-Chiang P.-H., Wang H.-C. , Lai Y.-L. , et al.Zoledronic acid treatment for cancerous bone metastases: a phase IV study in Taiwan J Cancer Res Ther, 9 (2013), pp. 653-659.

8-Van Poznak CH, Unger JM, Darke AK, et al. Association of Osteonecrosis of the Jaw With Zoledronic Acid Treatment for Bone Metastases in Patients With Cancer. JAMA Oncol. 2021;7(2):246-254. 
